\title{
Alcohols and Water as Reducing Agents in Radical Reactions
}

\author{
Davide Pozzi§ and Philippe Renaud* \\ $\S$ METTLER TOLEDO Award Winner (Oral Presentation)
}

\begin{abstract}
The radical reduction of B-alkylcatecholboranes into alkanes using alcohols and water as hydrogen atom source is described. This process involves a completely unexpected mechanism: the alcohol (or water) complexed to a borate derivative acts as a reducing agent. The complexation activates the alcohol resulting in a decrease of the $\mathrm{O}-\mathrm{H}$ bond dissociation energy. This approach offers promising opportunities for the development of tin-free radical reducing agent. Closely related reports from the recent literature involving boron and titanium derivatives are also presented.
\end{abstract}

Keywords: Alcohols · Bond dissociation energy · Organoboranes · Radicals · Reduction

\section{Introduction}

The reduction of alkyl radicals is a highly useful synthetic methodology. ${ }^{[1]}$ Tributyltin hydride $\left(n-\mathrm{Bu}_{3} \mathrm{SnH}\right)$ is the most versatile radical reducing agent thanks to its relatively weak, non-ionic bond between tin and hydrogen $(74 \mathrm{kcal} / \mathrm{mol})$, which can cleave homolytically. However organotin hydrides are toxic, expensive and difficult to remove from the desired products. ${ }^{[2-4]}$ These problems have therefore pushed research towards more environmentally benign metalfree reagents. A substantial effort has been made to replace stoichiometric tin in radical reductions. In addition various methods involving organosilanes, organogermanes and organophosphorus compounds have been developed. ${ }^{[5]}$ However, cheap, environmentally friendly, and versatile reagents for radical reductions are still needed.
In the course of our studies on the reduction of B-alkylcatecholboranes into alkanes, we disclosed that alcohols and water, complexed to an organoborane species, can reduce alkyl radicals and replace toxic tin hydrides as a hydrogen atom source in radical reactions. ${ }^{6]}$

B-Alkylcatecholboranes (= 2-alkyl1,2,3-benzodioxaborole) are easily obtained via hydroboration of olefins using catecholborane as the hydroborating agent ${ }^{[7-9]}$ (Scheme 1, reaction (1)). They undergo an efficient homolytic substitution at boron leading to primary, secondary, and tertiary alkyl radicals in an irreversible manner. ${ }^{[10]}$ It was demonstrated by ESR spectroscopy that the perboryl radical intermediate A resulting from the complexation of the alkoxyl radical with B-methylcatecholborane, is stabilized by delocalization onto the aromatic ring (Scheme 1, reaction (2)). ${ }^{[11]}$
The oxidative treatment of B-alkylcatecholboranes leading to the corresponding alcohol is a common transformation and the whole process represents an anti-Markovnikov addition of water to alkenes. ${ }^{[12]}$ On the other hand the reduction of B-alkylcatecholborane into the corresponding alkane is much less common. This transformation is usually achieved by treatment of a trialkylborane under severe conditions such as proprionic acid in diglyme at 162 ${ }^{\circ} \mathrm{C},{ }^{[13-15]}$ alkaline protonolysis ${ }^{[16,17]}$ and hydrogenolysis at high temperature (190-225 $\left.{ }^{\circ} \mathrm{C}\right) .[18,19]$

Since B-alkylcatecholboranes generate alkyl radicals under mild radical conditions, we decided to investigate their reduction. Moreover, during the allylation of $\alpha$-pinene (1) depicted in Scheme 2, we noticed the formation of a small amount $(\leq 5 \%)$ of cis-pinane (3). ${ }^{[20]}$ The presence of $\mathbf{3}$ was at-
${ }^{\star}$ Correspondence: Prof. P. Renaud

University of Bern

Department of Chemistry and Biochemistry

Freiestrasse 3

$\mathrm{CH}-3000$ Bern

Tel.: +41316314359

Fax: +41316313426

E-Mail: philippe.renaud@ioc.unibe.ch

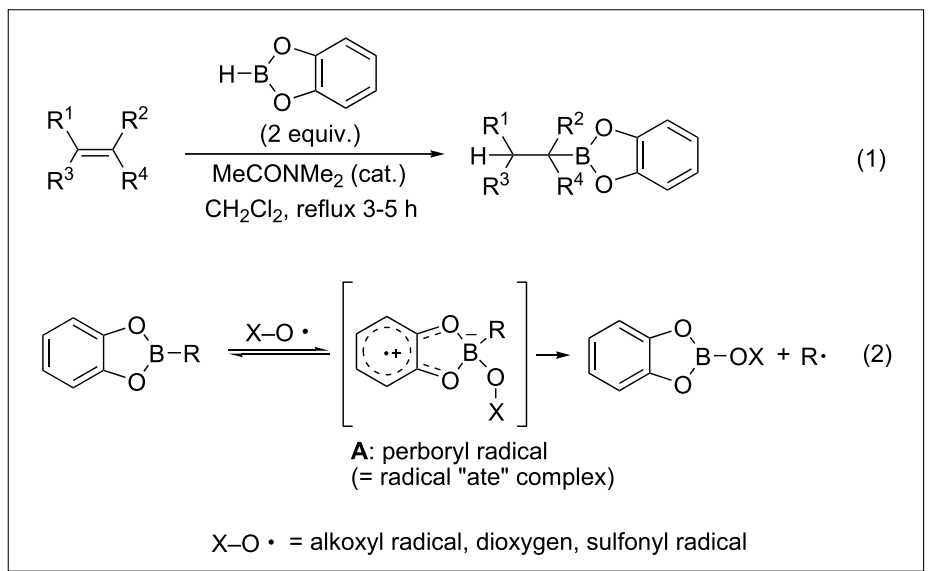

Scheme 1. B-Alkylcatecholboranes 


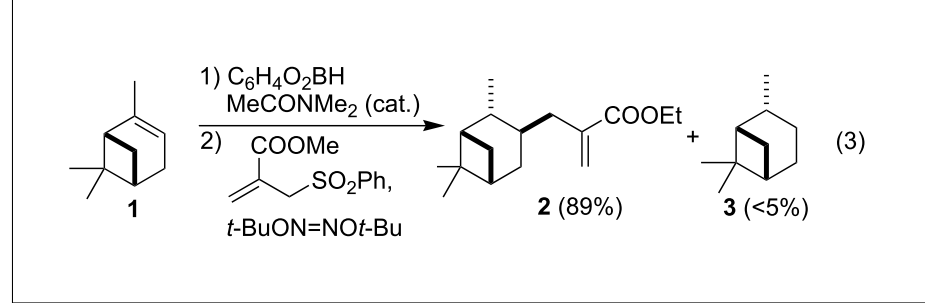

Scheme 2. Radical allylation of B-alkylcatecholboranes

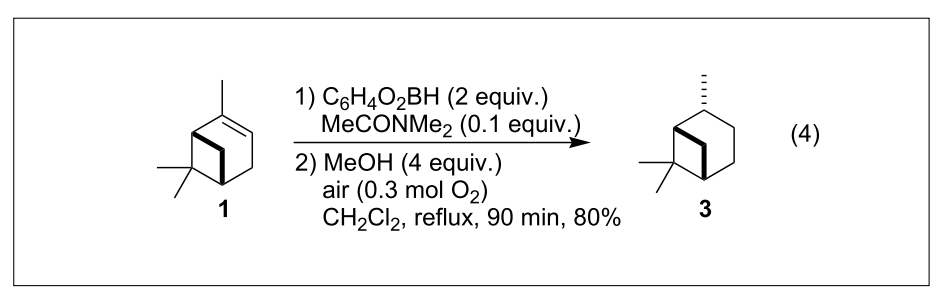

Scheme 3. Hydroboration-reduction of $\alpha$-pinene (1)

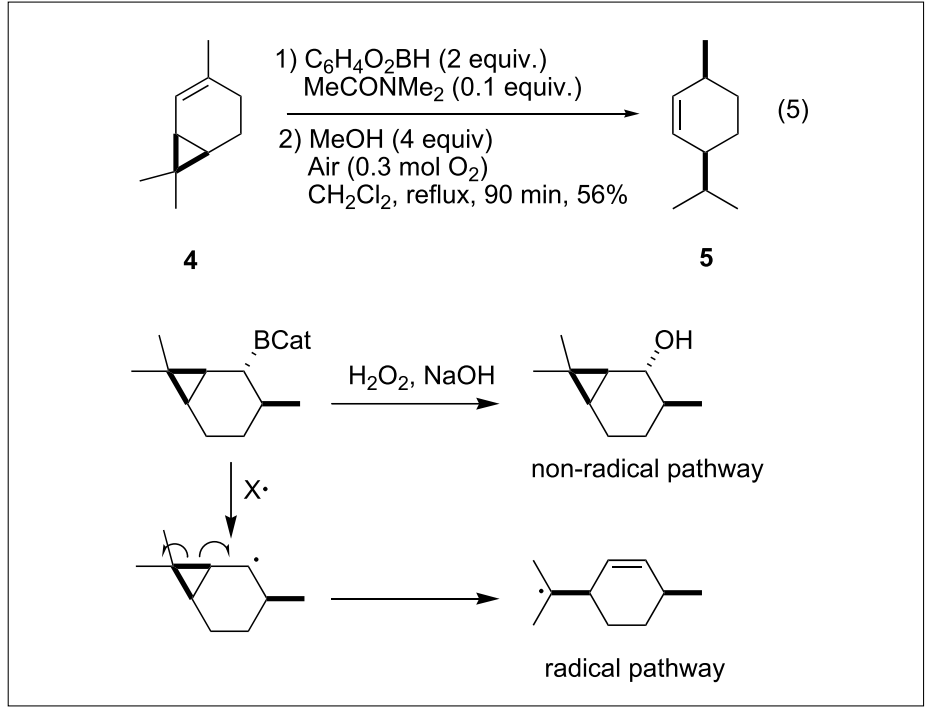

Scheme 4. Radical probe experiment with (+)-2-carene (4) tributed, without any experimental support, to partial protonation of the intermediate organoborane by the methanol used to decompose the excess of catecholborane. A careful investigation of this process led us to develop a mild method for the reduction of B-alkylcatecholborane. ${ }^{[6]}$ A very unusual use of alcohols and water as a reducing agent in a radical process is described.

\section{Results and Discussion}

Our investigation started with the reduction of the $\alpha$-pinene (1) into the corresponding alkane cis-pinane (3). The B-pinylcatecholborane was prepared in situ following the mild conditions developed by $\mathrm{Fu},{ }^{[21]}$ i.e. by treatment of $\alpha$-pinene with two equivalents of B-catecholborane and $10 \mathrm{~mol} \%$ of $\mathrm{N}, \mathrm{N}$-dimethylacetamide in refluxing dichloromethane. After completion of the hydroboration, the excess of the B-catecholborane was decomposed with four equivalents of $\mathrm{MeOH}$. Air ( 0.3 equiv. $\mathrm{O}_{2}$ ) was bubbled into the reaction mixture over a period of $90 \mathrm{~min}$. The formation of $\mathbf{3}$ was monitored by GC and a surprising $80 \%$ yield was observed after the addition of air (Scheme 3).

This observation prompted us to investigate the mechanism of the reaction in more detail. In order to discover the exact origin of the hydrogen atom, deuterium labeling experiments were run with $\alpha$-pinene. The B-pinylcatecholborane was prepared in situ under the same conditions described above and the reaction was initiated with air. The use of deuterated dichloromethane- $\mathrm{d}_{2}$ as solvent did not affect the reaction and the reduced compound $\mathbf{3}$ was obtained without deuterium incorporation. When the reaction was run with methanol- $\mathrm{d}_{3}\left(\mathrm{CD}_{3} \mathrm{OH}\right)$ the non-deuterated product 3 was obtained. Methanol-d $\mathrm{d}_{4}\left(\mathrm{CD}_{3} \mathrm{OD}\right)$ and methanol-d $\left(\mathrm{CH}_{3} \mathrm{OD}\right)$ gave the deuterated pinane with
$>80 \%$ deuterium incorporation and the reaction became very slow ( $17 \%$ yield after $90 \mathrm{~min}$ ). These results indicate that the transferred hydrogen atom comes from the $\mathrm{OH}$ bond of methanol. Similar results were obtained with $\mathrm{D}_{2} \mathrm{O}$.

Interestingly, the reaction of pure Bpinylcatecholborane, obtained via hydroboration of the $\alpha$-pinene (1) and purified by distillation, with four equivalents of methanol under oxygen initiation gave only traces of cis-pinane (3). These reaction conditions only differ from the ones we used initially by the presence of the excess of catecholborane used for the hydroboration. Adding one equivalent of catecholborane prior to the addition of $\mathrm{MeOH}$ gave the reduced product in $50 \%$ yield. This observation indicates that the presence of $\mathrm{MeO}-\mathrm{BO}_{2} \mathrm{C}_{6} \mathrm{H}_{4}$, resulting from the methanolysis of the unreacted catecholborane, is an essential component of the reaction.

The free-radical nature of the reaction had to be proved, since the homolytic bond dissociation of an $\mathrm{O}-\mathrm{H}$ bond from methanol or water are far too high (BDE $\mathrm{MeO}-\mathrm{H}=$ $105 \mathrm{kcal} / \mathrm{mol}, \mathrm{BDE} \mathrm{HO}-\mathrm{H}=116 \mathrm{kcal} / \mathrm{mol}$ ) to be involved directly as radical reducing agent. At this stage, an ionic pathway was not excluded. More acidic alcohols such as hexafluoroisopropanol, trifluoroethanol or 2-phenylethanol were tested. They gave the reduced product in less than $20 \%$ yield, showing that the acidity of the alcohol does not play an important role in the process.

The role of the radical initiator was then investigated. The B-pinylcatecholborane was prepared in situ under Fu's conditions. After the addition of $\mathrm{MeOH}$ (4 equiv.), the reaction was maintained at room temperature under nitrogen. The formation of the cis-pinane (3) was monitored by GC using an internal standard. After $90 \mathrm{~min}$, the reduced product was obtained in less than $10 \%$ yield. The conversion of the organobo- rane into the alkane was slow and $80 \%$ yield was obtained only after $60 \mathrm{~h}$. Furthermore under very strict oxygen exclusion (glovebox), the reduced product could not be detected after $60 \mathrm{~h}$. These results demonstrate that a radical initiator is required for the reduction.

A radical probe experiment was run using the monoterpene (+)-2-carene (4). The reduction afforded the monocyclic cis-para-menth-2-ene (5) in 56\% yield resulting from the ring opening of the cyclopropane ring suggesting that a free-radical intermediate is involved. Indeed, we have already established that an oxidative treatment with alkaline hydrogen peroxide leads to the corresponding alcohols without opening of the cyclopropane ring; in contrast a radical pathway generates a monocyclic product via a cyclopropylmethyl-homoallyl radical rearrangement (Scheme 4). ${ }^{[22]}$

A tentative mechanism is shown in Scheme 5. The alkyl radical is generated from the reaction between the B-alkylcatecholborane and a radical initiator (oxygen or an alkoxyl radical). The alkyl radical is then reduced by the complex $\mathbf{B}$ resulting from the coordination of the alcohol to the Lewis acidic $\mathrm{MeO}-\mathrm{BO}_{2} \mathrm{C}_{6} \mathrm{H}_{4}$. This complexation activates the alcohol producing a substantial decrease of the $\mathrm{O}-\mathrm{H}$ bond dissociation energy. The resulting radical $\mathbf{C}$ is closely related to the 'ate' complex A described in Scheme 1. It releases a molecule of MeO-BCat and a methoxyl radical which propagates the radical chain reaction. This mechanism fits with all the experimental observations. Various alcohols were tested: methanol, ethanol, isopropanol and water were found to work in a similar way. On the other hand phenylethanol and tert-butanol were found to be inefficient probably because of the rapid fragmentation of the alkoxyl radical intermediate leading to benzyl and methyl radicals, respectively. 


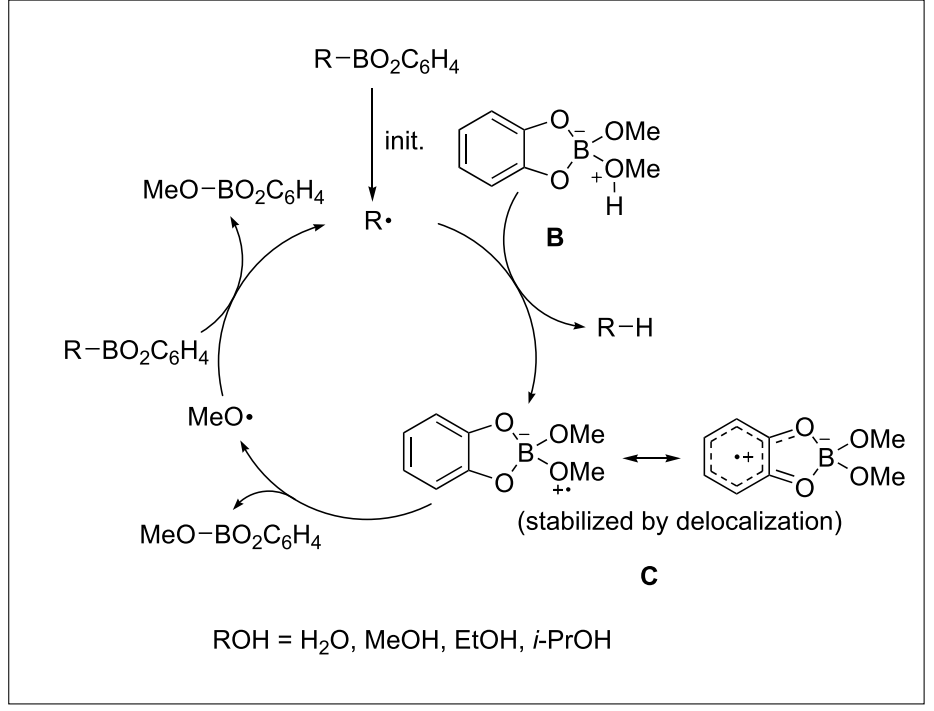

Scheme 5. Proposed mechanism for the reduction of B-alkylcatecholboranes

The scope and limitations of the reaction were investigated for a series of Balkylcatecholboranes (Scheme 6). Good yields were found with primary (reactions (6) and (7)), secondary (reactions (8)-(11)) and tertiary alkyl radicals (reaction (12)). Benzyl radicals are not reduced with this system indicating that the $\mathrm{O}-\mathrm{H}$ bond dissociation energy in complex B is not weak enough to reduce stabilized radicals.

\section{Related Results from the Literature}

\subsection{Triethylborane-Water Complex as a Reducing Agent}

A similar discovery was reported by Wood and coworkers while our manuscript

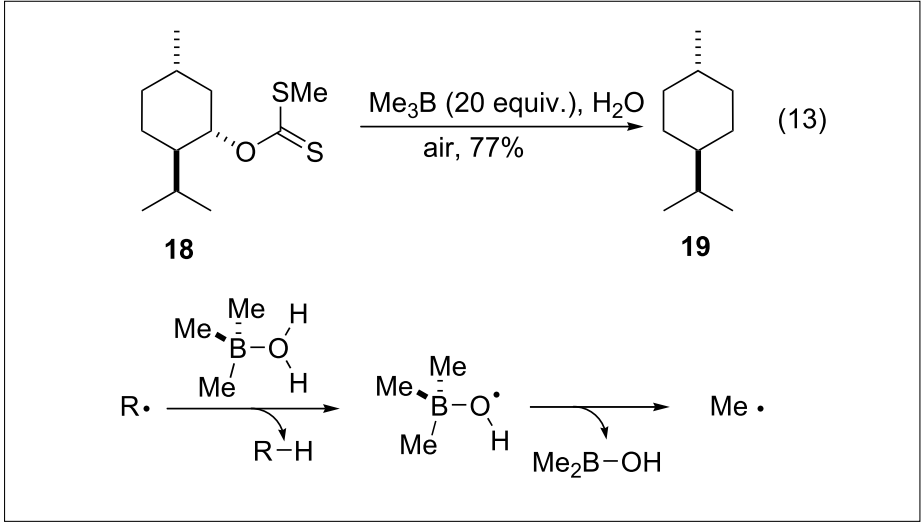

Scheme 7. Wood's modified Barton-McCombie reaction

Scheme 8. $\mathrm{TiCp}_{2} \mathrm{Cl}$-water induced reductive opening of epoxides

was in preparation. They disclosed that the system $\mathrm{H}_{2} \mathrm{O} / \mathrm{Et}_{3} \mathrm{~B}$ can be used as a source of hydrogen atom in the Barton-McCombie reaction (Scheme 7). ${ }^{[23]}$ Computational studies confirmed the lowered dissociation energy of water complexed to the $\mathrm{Me}_{3} \mathrm{~B}$ (86 $\mathrm{kcal} / \mathrm{mol}$ ) compared to that of the free water $(116 \mathrm{kcal} / \mathrm{mol})$.

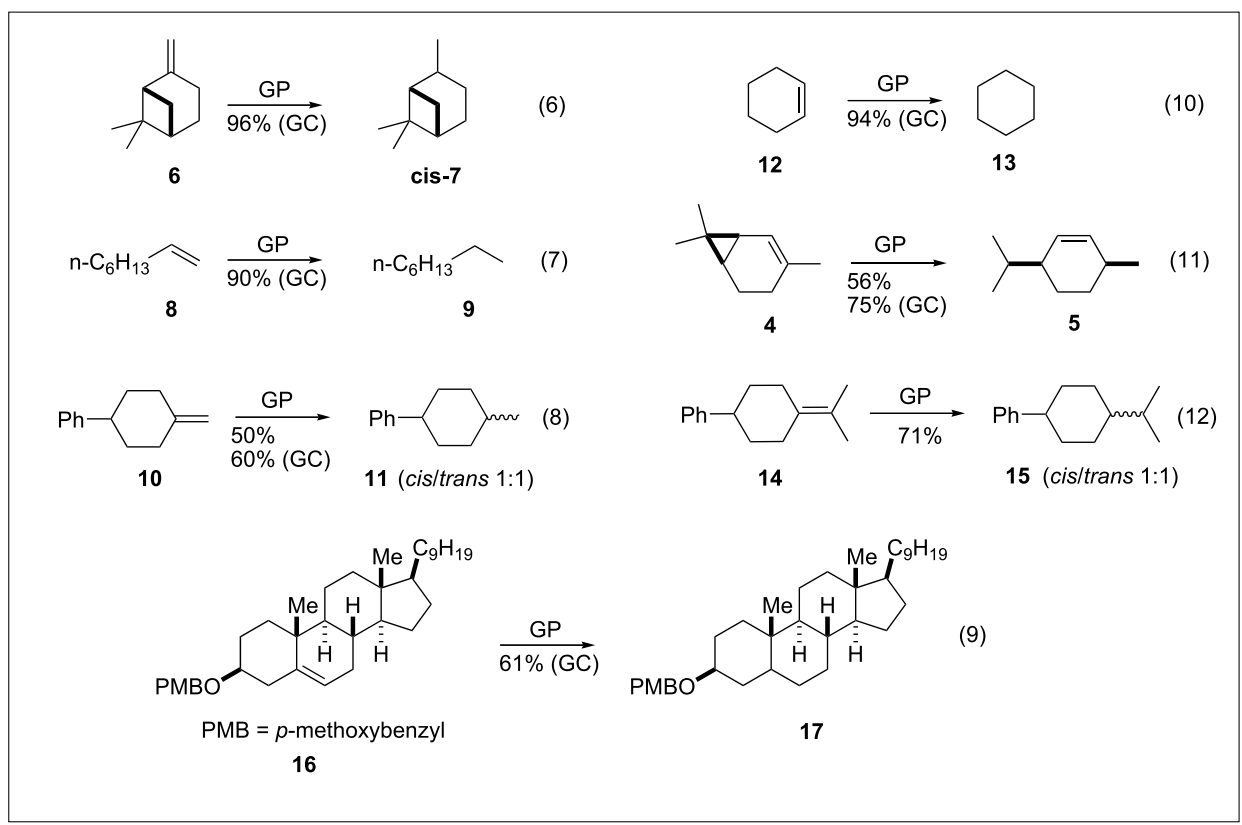

Scheme 6. General Procedure (GP): 1) $\mathrm{C}_{6} \mathrm{H}_{4} \mathrm{O}_{2} \mathrm{BH}$ (2 equiv.), $\mathrm{MeCONMe}{ }_{2}$ (0.1 equiv.) in $\mathrm{CH}_{2} \mathrm{Cl}_{2}$ 2) $\mathrm{MeOH}$ (4 equiv.), air (0.3 mol $\left.\mathrm{O}_{2}\right), \mathrm{CH}_{2} \mathrm{Cl}_{2}$, reflux, $90 \mathrm{~min}$

\subsection{Titanium(III)-Water as a Reducing Agent}

More recently, Oltra observed that during the $\mathrm{Cp}_{2}$ TiCl-mediated epoxide ring opening, the reduced product was obtained when water was added to the reaction mixture (Scheme 8). ${ }^{[24]}$ The mechanism of this reaction involves the aqua complex $\mathbf{2 0}$, which may act as a hydrogen atom donor by single electron transfer to the oxygen atom resulting in a Ti(IV) complex 21. Density functional theory calculations showed again a marked decrease of the $\mathrm{O}-\mathrm{H}$ homolytic bond dissociation energy when water is coordinated to the $\mathrm{Cp}_{2} \mathrm{Ti}^{\mathrm{III}} \mathrm{Cl}$ complex (49 $\mathrm{kcal} / \mathrm{mol}$ ).

\section{Conclusion}

In summary we have developed a new reaction for the reduction of B-alkylcatecholboranes into alkanes. This environmentally friendly methodology involves the use of alcohols and water as source of hydrogen atom under very mild conditions. To the best of our knowledge, this is the first radical-mediated reduction of organoboranes. These results, together with recent results from the literature, indicate that alcohols and water activated by Lewis acids may become valuable reducing agent in radical reactions offering substantial opportunities 
for the design of novel free-radical reducing agents to replace toxic tin hydride derivatives in radical processes. Recent progress in this field will be reported soon.

\section{Acknowledgments}

We are very grateful to the Swiss National Science Foundation (Grant 200020-112250) and to the University of Bern for financial support. We also thank BASF Corporation for the gift of catecholborane.

Received: March 13, 2007

[1] P. Renaud, M. P. Sibi, 'Radicals in Organic Synthesis', Wiley-VCH, Weinheim, 2001, and references cited therein.

[2] A. Buaguely, J. C. Walton, Angew. Chem., Int. Ed. 1998, 37, 3072.

[3] K. E. Appel, Drug. Metab. Rev. 2004, 36, 763.

[4] I. J. Boyer, Toxicology 1989, 55, 253.

[5] E. Dopp, L. M. Hartmann, A. M. Florea, A. W. Rettemeier, A. V. Hirner, Crit. Rev. Toxicol. 2004, 34, 301.

[6] D. Pozzi, E. M. Scanlan, P. Renaud, J. Am. Chem. Soc. 2005, 127, 14204.

[7] U. Wietelmann, Janssen Chimica Acta 1992, 10, 16.

[8] K. Burgess, M. J. Ohlmeyer, Chem. Rev. 1991, 91, 1179.

[9] G. W. Kabalka, Org. Prep. Proc. Int. 1977, 9, 131.

[10] A.-P. Schaffner, P. Renaud, Eur. J. Org. Chem. 2004, 2291.
[11] J. A. Baban, N. J. Goodchild, B. P. Roberts, J. Chem. Soc. Perkin Trans. 2 1986, 157.

[12] G. Zweifel, H. C. Brown, in 'Organic Reactions', Wiley, London, 1963, Vol. 13, p. 1.

[13] H. C. Brown, K. J. Murray, J. Am. Chem. Soc. 1959, 81, 4108.

[14] H. C. Brown, K. J. Murray, Tetrahedron 1986, 42, 5497.

[15] H. C. Brown, K. J. Murray, J. Org. Chem. 1961, 26, 631 .

[16] L. S. Vasilev, V. V. Veselovskii, B. M. Mikhailov, Bull. Acad. Sc. USSR Div. Chem. Sc. 1977, 26, 1031.

[17] P. R. Jones, T. R. O. Lim, J. Organomet. Chem. 1976, 120, 27.

[18] R. Köster, Angew. Chem. 1956, 68, 383.

[19] F. L. Ramp, E. J. Dewitt, L.E. Trapasso, J. Org. Chem. 1962, 27, 4368.

[20] A.-P. Schaffner, P. Renaud, Angew. Chem., Int. Ed. 2003, 42, 2658.

[21] C. E. Garett, G. C. Fu, J. Org. Chem. 1996, 61, 3224.

[22] C. Cadot, P. I. Dalko, J. Cossy, C. Ollivier, R. Chuard, P. Renaud, J. Org. Chem. 2002, 67, 7193.

[23] D. A. Spiegel, K. B. Wiberg, L. N. Schacherer M. R. Madeiros, J. L. Wood, J. Am. Chem. Soc. 2005, 127, 12513.

[24] J. M. Cuerva, A. C. Campagna, J. Justicia, A. Rosales, J. L. Oller-Lòpez, R. Robles, D. G. Càrdenas, E. Bunuel, E. J. Oltra, Angew. Chem., Int. Ed. 2006, 45, 5522. 